\title{
Estimativas de impacto da COVID-19 na mortalidade de idosos institucionalizados no Brasil
}

\author{
Estimates of the impact of COVID-19 \\ on mortality of institutionalized elderly in Brazil
}

\author{
Carla Jorge Machado (https://orcid.org/0000-0002-6871-0709) ${ }^{1}$ \\ Claudia Cristina de Aguiar Pereira (https://orcid.org/0000-0003-1389-9214) ${ }^{2}$ \\ Bernardo de Mattos Viana (https://orcid.org/0000-0001-8792-2996) ${ }^{3}$ \\ Graziella Lage Oliveira (https://orcid.org/0000-0002-3387-3583) ${ }^{1}$ \\ Daniel Carvalho Melo (https://orcid.org/0000-0001-7354-623X) ${ }^{4}$ \\ Jáder Freitas Maciel Garcia de Carvalho (https://orcid.org/0000-0002-1053-3236) ${ }^{5}$ \\ Flávia Lanna de Moraes (https://orcid.org/0000-0002-2638-0553) ${ }^{6}$ \\ Edgar Nunes de Moraes (https://orcid.org/0000-0002-8923-1029) ${ }^{7}$
}

${ }^{1}$ Departamento de

Medicina Preventiva

e Social, Faculdade de

Medicina, Universidade

Federal de Minas Gerais

(UFMG).

carlajmachado@gmail.com

${ }^{2}$ Departamento de

Administração e

Planejamento em Saúde,

Escola Nacional de Saúde

Pública Sergio Arouca,

Fiocruz. Rio de Janeiro RJ

Brasil.

${ }^{3}$ Departamento de Saúde

Mental, Faculdade de

Medicina, UFMG.

${ }^{4}$ MedLogic. Belo Horizonte

MG Brasil.

${ }^{5}$ Hospital das Clínicas,

UFMG. Belo Horizonte MG

Brasil.

${ }^{6}$ Secretaria Municipal de

Saúde, Prefeitura Municipal

de Belo Horizonte. Belo

Horizonte MG Brasil.

${ }^{7}$ Departamento de Clínica

Médica, UFMG. Belo

Horizonte MG Brasil.
Abstract The COVID-19 pandemic poses diffculties for long-term care institutions for the elderly, with increased mortality rates for the residents. This study aims to estimate the impact of COVID-19 on mortality of institutionalized elderly in Brazil. Estimates of the percentage of elderly deaths occurring in care homes were calculated for Brazil, States and Regions using estimates for the total number of deaths. The estimation was based upon information available for other countries. The weighted percentage was $44.7 \%$ and 107,538 COVID-19 deaths were estimated for the elderly in these institutions in Brazil in 2020. Higher numbers of deaths were expected in the Southeast Region (48,779 deaths), followed by the Northeast Region (28,451 deaths); São Paulo was the most affected State (24,500 deaths). The strong impact of COVID-19 on the elderly population living in long-term care facilities is clear. Estimates for the country exceeded 100,000 elderly people, potentially the most fragile and vulnerable, and are based upon a conservative number of total deaths, in view of other estimates and the alarming situation of death growth in Brazil from COVID-19. Key words Coronavirus infections, Simulation, Health of institutionalized elderly, Frail elderly, Cross-sectional studies
Resumo O presente estudo tem como objetivo estimar o impacto da COVID-19 na mortalidade de idosos institucionalizados no Brasil. Foram estimados números de óbitos pela doença para o País, Unidades da Federação e Regiões, com base nas estimativas calculadas e efetuadas neste trabalho do percentual de óbitos de idosos que ocorreriam em instituições de longa permanência de acordo com os totais. Essa estimativa foi baseada em informações disponiveis para uma série de países. $O$ percentual ponderado foi de 44,7\%. Estimaram-se 107.538 óbitos de idosos nestas instituiçães no Brasil em 2020, por COVID-19. São previstos maiores números de óbitos na Região Sudeste (48.779 óbitos), seguida da Região Nordeste (28.451 óbitos); São Paulo é a Unidade da Federação que na estimativa será mais afetada (24.500 óbitos). Fica claro o forte impacto da COVID-19 na população idosa residente em instituições de longa permanência para idosos. As estimativas ultrapassam para o país 100 mil idosos, potencialmente os mais frágeis e vulneráveis, e são baseadas em número de óbitos totais conservador, tendo em vista outras estimativas e a situação alarmante de crescimento dos números de óbitos no Brasil.

Palavras-chave Infecções por coronavírus, Simulação, Saúde do idoso institucionalizado, Idoso fragilizado, Estudos transversais 


\section{Introdução}

Altas letalidades por SARS-CoV-2 têm sido, em sua maior parte, associadas a pacientes idosos ou à presença de comorbidades mais comuns nestes pacientes ${ }^{1-3}$, sendo superiores a um quinto dos acometidos com mais de 80 anos, tanto na China $(21,9 \%)$ como na Itália $(20,2 \%)^{3}$. A pandemia de COVID-19 constitui um desafio para as instituições de longa permanência para idosos, dado o pertencimento dos institucionalizados aos grupos de maior susceptibilidade para a COVID-19 em suas formas mais graves, as quais podem resultar nos piores desfechos às infecções por SARS-CoV-2, incluindo o óbito ${ }^{4}$.

Instituições de longa permanência para idosos concentram pessoas com maior risco e, ademais, o fato de viverem muito próximas pode atuar como um agravante na propagação das infecções por SARS-CoV-2,3. Embora haja estimativas do total de óbitos de residentes de instituições de longa permanência para idosos em alguns países ${ }^{5,6}$, foram encontradas estimativas de letalidade da COVID-19 em idosos institucionalizados apenas nos Estados Unidos e em Cingapura. Nos Estados Unidos (Condado de King, estado de Washington $)^{4}$ a letalidade foi de 33,7\% e, em Cingapura ${ }^{7}$, a estimativa é indireta, mas indica que óbitos de idosos institucionalizados pela COVID-19 responderam por cerca de $14 \%$ da letalidade em todas as idades ${ }^{7}$.

Existem esforços em alguns países de se estimar o impacto da mortalidade ${ }^{5,6}$, porém, no Brasil não se sabe ao certo o número de idosos residentes em instituições de longa permanência nem em que medida a COVID-19 tem afetado a mortalidade dos residentes nestes estabelecimentos. Assim, dada a lacuna no conhecimento da mortalidade essa população idosa institucionalizada, o presente estudo tem como objetivo estimar o impacto da COVID-19 na mortalidade de idosos institucionalizados no Brasil.

\section{Método}

Trata-se de estudo transversal e de simulação. As estimativas apresentadas neste trabalho foram calculadas em duas etapas. Na primeira etapa, estimaram-se os óbitos totais em idosos por COVID-19 e na segunda, aplicou-se a esses óbitos uma porcentagem ponderada obtida com base em indicadores de mortalidade proporcional advindas de dados oficiais de óbitos em instituições de longa permanência para idosos de diferentes países - como China, Itália e Coréia do Sul - ao Brasil. A mortalidade proporcional se constitui, nesse caso, nos óbitos residentes em care homes por COVID-19 em relação aos óbitos totais em cada uma das diferentes localidades.

Em relação à primeira fase das estimativas, os óbitos totais em idosos por COVID-19 provieram da estimativa mínima sugerida por Martinez et al. ${ }^{8}$, que utilizaram a plataforma Corona-older 9 . Esta plataforma permite estimar o número de mortes esperadas por COVID-19, por idade, para o Brasil, Unidades da Federação (estados e Distrito Federal), a partir de estimativas populacionais de 2020. Permite, ainda, que pesquisadores trabalhem com taxas variáveis de infecção e letalidade de casos baseadas no que aconteceu em países como China, Itália ou Coreia do Sul. A opção, neste trabalho, em se trabalhar com base nas estimativas de Martinez et al. ${ }^{8}$, que selecionaram uma taxa de infecção de $10,0 \%$ e taxas de letalidade dos casos por grupos etários observadas na China, obtendo-se um número de óbitos totais de 240.281, foi considerada razoável e conservadora frente à estimativa de 452.694 de Dowd et al. ${ }^{10}$, que verificaram, em detalhe, não apenas o comportamento da epidemia, mas também a estrutura etária do Brasil.

Em relação à segunda fase das estimativas, que sucedeu à definição do número de óbitos por COVID-19, com o objetivo de realizar as estimativas de óbitos em instituições de longa permanência para idosos no Brasil, utilizaram-se dados de pesquisas internacionais recentes sobre COVID-19 com dados oficiais de onze diferentes países, em datas próximas, publicadas por Comas -Herrera et al. ${ }^{5}$. As estimativas, acompanhadas de sua localização temporal e geográfica ${ }^{5}$, estão dispostas na Tabela 1.

Quanto aos dados da Tabela 1, ressalte-se: em primeiro lugar, utilizaram-se, sempre que possível, estimativas de óbitos confirmados, por COVID-19, para o país - essa escolha foi feita para evitar incluir indivíduos falso-positivos para COVID-19 que vieram a óbito posteriormente (Bélgica e Irlanda teriam, caso fossem utilizados os dados de óbitos de pessoas por causa COVID-19 como provável, $100,0 \%$ e $56,9 \%$, nas mesmas datas apresentadas na Tabela 1); em segundo lugar, foi obtida informação para os Estados Unidos com base em dados de 38 dos 50 estados norte -americanos ${ }^{6}$; finalmente, a informação para a Espanha foi obtida com base nas 6 das 17 comunidades autônomas e o percentual foi calculado diretamente pelos autores com base nas informações disponibilizadas em Comas-Herrera et al. ${ }^{5}$. 
Tabela 1. Informações utilizadas para as estimativas do percentual ponderado de óbitos de idosos em instituições de longa permanência para idosos no Brasil, com base em estudos de Comas-Herrera et al. ${ }^{5}$ e Chidambaram ${ }^{6}$.

\begin{tabular}{|c|c|c|c|c|}
\hline País & $\begin{array}{c}\text { Datas } \\
\text { referentes às } \\
\text { informações de } \\
\text { ocorrência do } \\
\text { óbito } \\
\text { (dia/mês/ano) }\end{array}$ & $\begin{array}{c}\text { (N1) } \\
\text { Número de } \\
\text { óbitos por } \\
\text { COVID-19 } \\
\text { nos países } \\
\text { com } \\
\text { informação }\end{array}$ & $\begin{array}{c}(\mathrm{N} 2) \\
\text { Número de óbitos } \\
\text { de residentes } \\
\text { de care home } \\
\text { por COVID-19 } \\
\text { nos países com } \\
\text { informação }\end{array}$ & $\begin{array}{c}(\mathrm{N} 3)=(\mathrm{N} 2) /(\mathrm{N} 1)(\mathrm{em} \%) \\
\text { Proporção de óbitos } \\
\text { de residentes de care } \\
\text { home por COVID-19 } \\
\text { em relação ao número } \\
\text { de óbitos totais por } \\
\text { COVID-19* }\end{array}$ \\
\hline Austrália & 25/abril/2020 & 80 & 15 & $19,0 \%$ \\
\hline Bélgica & 26/abril/2020 & 3.685 & 373 & $10,0 \%$ \\
\hline Canadá & $25 /$ abril/2020 & 2.465 & 1.769 & $72,0 \%$ \\
\hline França & $25 /$ abril/2020 & 22.614 & 8.564 & $51,0 \%$ \\
\hline Hungria & $18 /$ abril/2020 & 172 & 33 & $19,0 \%$ \\
\hline Irlanda & 24/abril/2020 & 829 & 452 & $55,0 \%$ \\
\hline Israel & 26/abril/2020 & 200 & 65 & $33,0 \%$ \\
\hline Noruega & $25 /$ abril/2020 & 193 & 122 & $63,0 \%$ \\
\hline Portugal & 23/abril/2020 & 820 & 327 & $40,0 \%$ \\
\hline Singapura & $16 /$ abril/2020 & 10 & 2 & $20,0 \%$ \\
\hline Espanha $^{(2)}$ & 22 ou 23/abril/20 & 19.580 & 9.789 & $50,0 \%$ \\
\hline Estados Unidos da América & 23/abril/2020 & 10.378 & 2.386 & $27,0 \%$ \\
\hline $\begin{array}{l}\text { Total e estimativa para o } \\
\text { Brasil }\end{array}$ & ---- & 61.026 & $23.897^{\star}$ & $44,7 \% *$ \\
\hline
\end{tabular}

Nota: ${ }^{*}$ para os totais (N2) e (N3) foram calculadas médias ponderadas por (N1).

O percentual ponderado de óbitos de residentes de care homes em relação ao total foi estimado como o somatório do número de óbitos por COVID-19 nos países com informação multiplicado pela proporção de óbitos de residentes de care homes por COVID-19 (N1) em relação ao número de óbitos totais por COVID-19, para todos os países (N3), divido pela soma de (N1) para todos os países:

$$
\begin{gathered}
\text { Percentual Ponderado }= \\
{[(80) \mathrm{x}(19,0)+(3.685) \mathrm{x}(10,0)+(2.465)} \\
\mathrm{x}(72,0)+(22.614) \mathrm{x}(51,0)+(172) \mathrm{x}(19,0)+(829) \\
\mathrm{x}(55,0)+(200) \mathrm{x}(33,0)+(193) \mathrm{x}(63,0)+(820) \\
\mathrm{x}(40,0)+(10) \mathrm{x}(20,0)+(19.580) \mathrm{x}(50,0)+(10.378) \\
\mathrm{x}(27,0)] /(80+3.685+2.465+22.614+172+829+20 \\
0+193+820+10+19.580+10.378)=44,7
\end{gathered}
$$

Foram obtidos intervalos de 95,0\% de confiança para o número de óbitos por COVID-19 estimados para idosos institucionalizados.

\section{Resultados}

$\mathrm{Na}$ Tabela 2 estão apresentadas as estimativas por Unidades da Federação, Regiões e para o Brasil.
Dos 240.287 óbitos por COVID-19 considerados, ocorreriam em instituições de longa permanência para idosos um total de 107.528 mortes em idosos nestas instituições (intervalo de 95,0\% de confiança de 107.269 a 107.788). Esses óbitos perfazem $61,9 \%$ de todos os 175.435 óbitos por COVID-19 em idosos. A Região Sudeste concentraria a maior parte dos óbitos do Brasil, equivalendo a 48.779 mortes (intervalo de $95,0 \%$ de confiança de 48.661 a 48.898), seguida de Região Nordeste, que teve 28.451 mortes (intervalo de $95,0 \%$ de confiança de 28.382 a 28.520 ), conforme a Figura 1.

Em relação às Unidades da Federação, observa-se que São Paulo, Minas Gerais, Rio de Janeiro, Bahia e Rio Grande do Sul, seriam os estados com o maior número de óbitos por COVID-19, tanto óbitos em todas as idades, quanto de idosos institucionalizados (Figura 2).

\section{Discussão}

Os achados do presente estudo indicaram que quase a metade $(44,7 \%)$ dos óbitos por COVID-19 ocorrerá entre os idosos institucionalizados e que mais de 100 mil idosos (107.528 óbitos) 
Tabela 2. Número de óbitos por COVID-19 estimados em idosos vivendo em instituições de longa permanência para idosos, para Brasil, Regiões, e Unidades da Federação, 2020.

\begin{tabular}{|c|c|c|c|c|}
\hline \multirow{2}{*}{$\begin{array}{c}\text { Estados/Distrito } \\
\text { Federal/Regiões/ } \\
\text { Brasil }\end{array}$} & \multirow{2}{*}{$\begin{array}{c}\text { (N1) } \\
\text { Número total de } \\
\text { óbitos (todas as } \\
\text { idades) considerados } \\
\text { por COVID-19 }\end{array}$} & \multirow{2}{*}{$\begin{array}{c}(\mathrm{N} 2)=(\mathrm{N} 1)^{\star} 0,447 \\
\text { Número Estimado de } \\
\text { Óbitos em instituições } \\
\text { de longa permanência } \\
\text { para idosos }\end{array}$} & \multicolumn{2}{|c|}{ Intervalo de $95 \%$ de Confiança } \\
\hline & & & limite inferior & limite superior \\
\hline Rondônia & 1.516 & 678 & 677 & 680 \\
\hline Acre & 640 & 286 & 286 & 287 \\
\hline Amazonas & 2.917 & 1.305 & 1.302 & 1.309 \\
\hline Roraima & 366 & 164 & 163 & 164 \\
\hline Pará & 7.092 & 3.174 & 3.166 & 3.181 \\
\hline Amapá & 522 & 234 & 233 & 234 \\
\hline Tocantins & 1.481 & 663 & 661 & 664 \\
\hline Maranhão & 6.921 & 3.097 & 3.090 & 3.105 \\
\hline Piauí & 3.897 & 1.743 & 1.740 & 1.748 \\
\hline Ceará & 10.636 & 4.760 & 4.748 & 4.771 \\
\hline Rio Grande do Norte & 4.022 & 1.800 & 1.796 & 1.804 \\
\hline Paraíba & 4.969 & 2.224 & 2.218 & 2.229 \\
\hline Pernambuco & 10.530 & 4.712 & 4.701 & 4.723 \\
\hline Alagoas & 3.316 & 1.484 & 1.480 & 1.487 \\
\hline Sergipe & 2.275 & 1.018 & 1.016 & 1.021 \\
\hline Bahia & 17.012 & 7.613 & 7.595 & 7.631 \\
\hline Minas Gerais & 27.024 & 12.093 & 12.064 & 12.122 \\
\hline Espírito Santo & 4.471 & 2.001 & 1.996 & 2.006 \\
\hline Rio de Janeiro & 22.912 & 10.253 & 10.228 & 10.278 \\
\hline São Paulo & 54.748 & 24.500 & 24.441 & 24.556 \\
\hline Paraná & 13.384 & 5.990 & 5.975 & 6.004 \\
\hline Santa Catarina & 8.040 & 3.598 & 3.589 & 3.607 \\
\hline Rio Grande do Sul & 15.960 & 7.142 & 7.125 & 7.159 \\
\hline Mato Grosso do Sul & 2.928 & 1.310 & 1.307 & 1.313 \\
\hline Mato Grosso & 3.206 & 1.434 & 1.431 & 1.438 \\
\hline Goiás & 6.947 & 3.109 & 3.101 & 3.116 \\
\hline Distrito Federal & 2.556 & 1.143 & 1.141 & 1.147 \\
\hline \multicolumn{5}{|l|}{ Regiões } \\
\hline Norte & 14.533 & 6.504 & 6.488 & 6.519 \\
\hline Nordeste & 63.578 & 28.451 & 28.382 & 28.520 \\
\hline Sudeste & 109.156 & 48.779 & 48.661 & 48.898 \\
\hline Sul & 37.384 & 16.729 & 16.689 & 16.790 \\
\hline Centro-Oeste & 15.636 & 6.997 & 6.980 & 7.014 \\
\hline Brasil & 240.287 & 107.528 & 107.269 & 107.788 \\
\hline
\end{tabular}

serão fatalmente atingidos no País. Mais do que as estimativas em si, que podem mudar e, desejavelmente, diminuírem caso haja políticas destinadas para tal, chamar a atenção para a questão dos idosos em instituições de longa permanência é fundamental.

As limitações do estudo podem ter afetado os resultados, pois a estimativa de óbitos por COVID-19 em idosos institucionalizados não con- siderou a estrutura etária ou a oferta de vagas nestas instituições por Unidades da Federação. Assim, esperar-se-ia maior número de óbitos em instituições de longa permanência para idosos na Região Sudeste pelo maior contingente populacional, elevada proporção de idosos $(16,1 \%)$ em relação à população total ${ }^{11}$, pelo percentual mais elevado de idosos institucionalizados e, finalmente, devido ao número de óbitos totais 


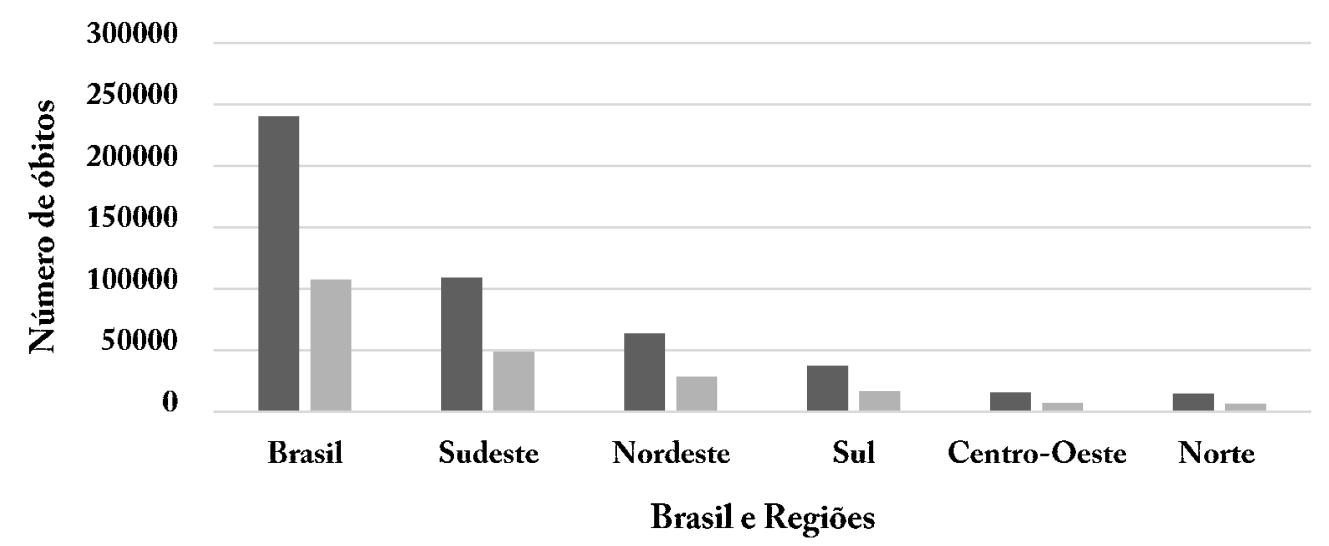

óbitos totais por COVID-19

óbitospor COVID-19 de idosos residentes em insituições de longa permanência para idosos

Figura 1. Número de óbitos por COVID-19 para todas as idades e para idosos vivendo em instituições de longa permanência para idosos, para Brasil e Regiões, 2020.

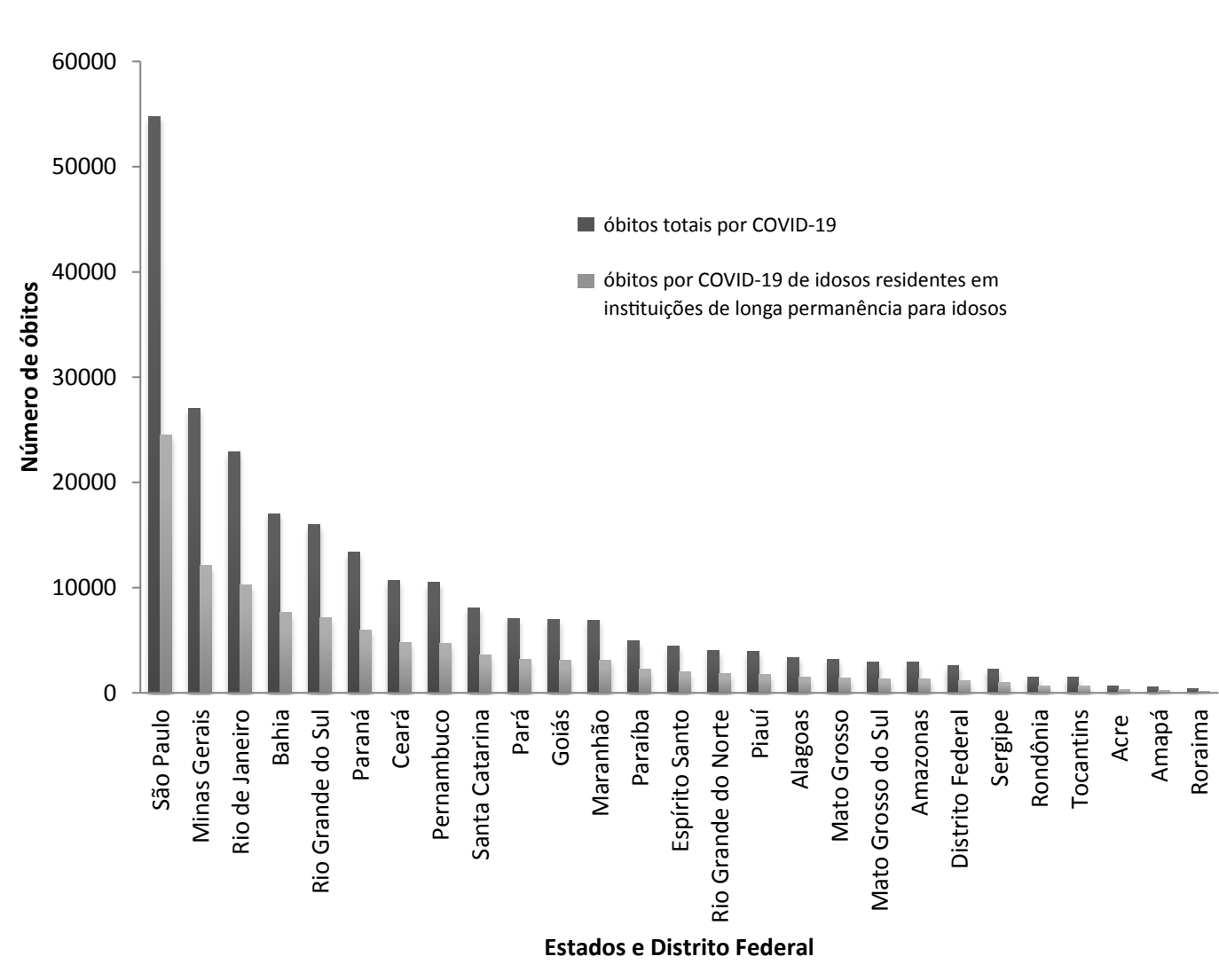

Figura 2. Número de óbitos por COVID-19 para todas as idades e para idosos vivendo em instituições de longa permanência para idosos, para Estados e Distrito Federal, 2020. 
por COVID-19 que vem sendo observado nas últimas semanas epidemiológicas na Região ${ }^{12}$. Ao longo do tempo, serão necessários ajustamentos nas estimativas para aferir se as estimativas iniciais estariam em concordância com o que de fato vem sendo verificado. Para tanto, são necessários também estudos específicos com idosos institucionalizados no Brasil no que se refere à letalidade por COVID-19.

Outra limitação se refere à própria indisponibilidade de dados sobre o quantitativo de idosos residentes em instituições e de estatísticas oficiais sobre a mortalidade nestes estabelecimentos, o que tornou inviável e pouco mais que especulativa a tentativa de calcular taxas de mortalidade para esta população. Com efeito, dados precisos sobre residentes em instituições de longa permanência para idosos são escassos e países e regiões como o Reino Unido também trabalham com estimativas $^{13}$. Tendo em vista essa dificuldade, nossa opção, portanto, foi estimar apenas o número de óbitos.

As simulações apontam para o impacto devastador da COVID-19 em idosos institucionalizados, assim como ocorreu em países como Itá$\operatorname{lia}^{14}$ e Estados Unidos ${ }^{4}$.

Cabrero $^{15}$, com base em experiência na Espanha, destaca haver causas para o impacto da pandemia por COVID-19 em idosos institucionalizados, quais sejam, negligência e discriminação causada em parte pela sobrecarga da pandemia nos serviços de saúde; insuficiência de recursos humanos e materiais; falta de controle e legislação voltados para estes idosos no início da crise. Assim, recomendam-se medidas que promovam a redução de riscos como restrição de visitantes às instituições; triagem para identificar sintomas em qualquer indivíduo que necessite entrar; uso de equipamentos de proteção individual e ampliação da testagem nesses estabelecimentos. Essas medidas foram implementadas em Cingapura $^{7}$ que, embora tenha tido casos e mesmo óbitos, tiveram sucesso na contenção de surtos por COVID-19 em idosos institucionalizados?

Discriminação e estigma de idosos institucionalizados correm o risco de acontecer e essa também deve ser uma preocupação dos formuladores de políticas. Documento das Nações Unidas, datado de maio de $2020^{16}$, chama a atenção para o discurso público e em mídias sociais, ressaltando que nesses tempos da pandemia por COVID-19, expressões de ressentimento intergeracional têm ocorrido. Políticas, programas e comunicações devem fornecer imagem diferenciada, mas não distorcida, do impacto da pandemia nos idosos, promovendo o envolvimento mais amplo da comunidade com a questão dos idosos, seus riscos diferenciados e, especialmente, chamando a atenção para aqueles institucionalizados. Essa atitude programática pode promover solidariedade intergeracional, fundamental para a união entre as pessoas, especialmente em momentos de crise $^{16}$.

Os achados da presente pesquisa podem ser utilizados por outros estudiosos para que possam efetuar estimativas de número de óbitos por COVID-19 em idosos em outros países. Tais simulações são extremamente importantes em momentos críticos como o presente, onde há escassez de informações diretas, trazendo a possibilidade de nortear o planejamento de ações de políticas públicas. 


\section{Colaboradores}

CJ Machado e CCA Pereira: concepção do artigo; análise de dados; escreveram o artigo; leram e aprovaram a versão final. BM Viana: concepção do artigo; auxílio com análise de dados; escreveu o artigo; leu e aprovou a versão final. GL Oliveira e DC Melo: auxílio com análise de dados; escreveram o artigo; leram e aprovaram a versão final. JFMG Carvalho e FL Moraes: escreveram o artigo; leram e aprovaram a versão final. EM Moraes: coordenador do estudo principal com idosos institucionalizados; concepção do artigo; escreveu o artigo; leu e aprovou a versão final.

\section{Referências}

1. Barra RP, EN Moraes EN, Jardim AA, Oliveira KK, Bonatti PCR, Issa AC. A importância da gestão correta da condição crônica na Atenção Primária à Saúde para o enfrentamento da COVID-19 em Uberlândia, Minas Gerais. APS em Revista 2020; 2(1):38-43.

2. Lloyd-Sherlock P, Ebrahim S, Geffen L, McKee M. Bearing the brunt of covid-19: older people in low and middle income countries. BMJ 2020; 368:m 1052 [acessado 2020 Abr 30]. Disponível em: https://www. bmj.com/content/368/bmj.m1052

3. Lai CC, Wang JH, Ko WC, Yen MY, Lu MC, Lee CM, Hsueh PR; Society of Taiwan Long-term Care Infection Prevention and Control. COVID-19 in long-term care facilities: An upcoming threat that cannot be ignored. J Microbiol Immunol Infect 2020; 53(3):444446.

4. D'Adamo H, Yoshikawa T, Ouslander JG. Coronavirus Disease 2019 in Geriatrics and Long-Term Care: The ABCDs of COVID-19. J Am Geriatr Soc 2020; 68(5):912-917.

5. Comas-Herrera A, Zalakain J, Litwin C, Hsu A, Fernandez-Plotka J. Mortality associated with COVID-19 outbreaks in care homes: early international evidence. [acessado 2020 Abr 24]. Disponível em: https://ltccovid.org/wp-content/uploads/2020/05/Mortality-associated-with-COVID-26-April-1.pdf

6. Chidambaram P. State Reporting of Cases and Deaths Due to COVID-19 in Long-Term Care Facilities. [acessado 2020 Abr 24]. Disponível em: https://www. kff.org/medicaid/issue-brief/state-reporting-of-cases-and-deaths-due-to-covid-19-in-long-term-carefacilities/

7. Tan L, Seetharaman S. COVID-19 outbreak in nursing homes in Singapore. J Microbiol Immunol Infect [acessado 2020 Jun 16]. Disponível em: https://doi. org/10.1016/j.jmii.2020.04.018

8. Martinez R, Loyd-Sherlock P, Souza LE, Giacomin K. Estimativas do impacto do Covid-19 na mortalidade no Brasil. [acessado 2020 Abr 11]. Disponível em: https://www.abrasco.org.br/site/outras-noticias/ saude-da-populacao/estimativas-do-impacto-da- covid19-na-mortalidade-no-brasil/46151/

9. Corona-older.com. Information and tools about coronavirus and older people in developing countries. [acessado 2020 Abr 11]. Disponível em: https://www. corona-older.com/tool

10. Dowd JB, Andriano L, Brazel DM, Rotondi V, Block P, Ding X, Liu Y, Mills MC. Demographic science aids in understanding the spread and fatality rates of COVID-19. [acessado 2020 Jun 11]. Disponível em: https://www.pnas.org/content/pnas/early/2020/04/15/2004911117.full.pdf

11. Instituto Brasileiro de Geografia e Estatística (IBGE). Censo demográfico 2010. Projeções da população [Internet]. IBGE; 2011. [acessado 2020 Jun 17]. Disponível em: https://www.ibge.gov.br/estatisticas/sociais/ populacao/9109-projecao-da-populacao.html?=\&$\mathrm{t}=$ resultados

12. Brasil.io. Covid-19. 2020 [acessado 2020 Jun 16]. Disponível em: https://brasil.io/dataset/covid19/caso_ full/ 
13. How big is the problem in care homes? $B B C$ news 2020 Abr 30; [acessado 2020 Maio 10]. Disponível em: https://www.bbc.com/news/health-52284281

14. Parodi E. Uncounted among coronavirus victims, deaths sweep through Italy's nursing homes. Reuters 2020 Mar 18; [acessado 2020 Jun 16]. Disponível em: https://www.reuters.com/article/us-health-coronavirus-italy-homes-insigh/uncounted-among-coronavirus-victims-deaths-sweep-through-italys-nursing-homes-idUSKBN2152V0

15. Cabrero GR. La crisis del coronavirus y su impacto en las residencias de personas mayores en España. Cien Saude Colet 2020; 25(6):1996.

16. United Nations (UN). Policy Brief: The Impact of COVID-19 on older persons. 2020. [acessado 2020 Maio 10]. Disponível em: https://unsdg.un.org/sites/default/files/2020-05/Policy-Brief-The-Impact-of-COVID-19-on-Older-Persons.pdf

Artigo apresentado em 12/05/2020

Aprovado em 26/06/2020

Versão final apresentada em 28/06/2020 